\title{
EDITORIAL
}

\section{Gut Microbiome in HIV Infection: Overcoming Barriers?}

\author{
Brett Williams ${ }^{1}$
}

Published online: 6 February 2019

(c) Springer Science+Business Media, LLC, part of Springer Nature 2019

The care of individuals infected with the human immunodeficiency virus (HIV) has remarkably improved in the last two decades, with the majority of patients receiving care enjoying health comparable, but not equivalent to that of their uninfected peers [1]. Despite these advances, HIV-infected, but virally suppressed individuals disproportionately suffer from the diseases of aging [1]. Over the last 10 years, much of the research regarding this disproportionate risk has focused on the gut as a source of chronic immune activation and inflammation, driving early-onset age-related morbidity and mortality. Individuals infected with HIV have elevated serologic markers of gut microbial translocation such as lipopolysaccharide (LPS), soluble CD14 (sCD14), and intestinal fatty acid-binding protein (I-FABP) that are thought to cause or contribute to the chronic immune activation characteristic of HIV. A strong link has been established among serologic markers of gut barrier integrity including intestinal fatty acid-binding protein (I-FABP), chronic immune activation, and disproportionate morbidity and mortality in HIV [2]. Several mechanisms have been proposed to explain this presumed gut-derived chronic inflammatory response, including altered composition or metabolic activity of the gut microbiota as well as increased gut epithelial permeability or "leaky gut." Though only fairly limited histologic data exist to date, it is generally accepted that gut-associated lymphoid tissue is severely damaged early in the course of HIV infection and that this damage is not entirely ameliorated by effective antiretroviral therapy [3].

Studies of gut microbial composition in HIV-infected individuals have been limited by relatively small cohorts and confounding factors which likely also influence the gut microbiota of HIV-negative controls. Geography, age, diet, and sexual orientation all appear to substantially contribute to the composition of the gut microbiome [4]. Given the

Brett Williams

Brett_Williams@Rush.edu

1 Rush University Infectious Disease, 600 S. Paulina \#143, Chicago, IL, USA potential for confounding variables, it is not surprising that few studies have found comparable HIV-associated alterations in gut microbial composition [5]. Although the most robust associations between gut microbial composition and HIV status appear to be a relative increase in the Prevotella/ Bacteroides ratio and an increase in Proteobacteria [5], the altered Prevotella/Bacteroides ratio appears to be driven primarily by host sexual orientation [4].

Fermentative gut microbes produce short-chain fatty acids (SCFAs), which include butyrate. SCFAs are important for maintaining the health of gut epithelial cells and modulating local immune responses [5]. Butyrate is hypothesized to work as a histone deacetylase inhibitor (HDACi), ultimately leading to increased expression of tight junction proteins such as claudin-1 [6]. Butyrate may also reduce gut inflammation by inducing regulatory $\mathrm{T}$ cells (Tregs) and modulating activation of antigen-presenting cells [7] SCFA enemas, particularly butyrate, have successfully treated ulcerative colitis in humans, though clinical trial results are not uniformly positive [8]. Although prior studies have found that butyrate-producing bacteria are selectively reduced in stool samples from HIV-infected persons, to date very few have reported directly measured stool SCFA levels $[4,7]$. Serrano-Villar et al. [9] did find that HIV-infected individuals had a distinct SCFA profile in stool compared to HIV-negative controls, with increased propionate and lower levels of acetate. Though several genera of butyrateproducing bacteria were underrepresented in HIV-infected individuals, this did not translate into baseline differences in stool butyrate levels. In the same study, a prebiotic intervention did increase abundance of several butyrate-producing bacteria in HIV-infected individuals, which correlated with increased stool butyrate levels, and decreases in soluble markers of immune activation.

In this issue of Digestive Diseases and Sciences, Qing et al. [10] report a cross-sectional comparison of 15 asymptomatic HIV-infected men who have sex with men treated with non-nucleoside reverse transcriptase inhibitors (NNRTI)-based combination antiretroviral therapy with 10 non-matched HIV-negative heterosexual male controls. The 
highest viral load noted was 260 and the majority had cluster of differentiation (CD), 4 counts of $200-500$ cells $/ \mu \mathrm{L}$ (normal 395-1651 cells $/ \mu \mathrm{L}$ ), consistent with at least reasonable HIV viral control. Data include 16S metagenomic analysis of the stool microbiome, gas chromatography-mass spectrophotometric (GC-MS) analysis of stool SCFA concentrations, and routine histology and immunohistochemistry for the intercellular junction protein claudin of colonic biopsies. Peripheral blood samples were analyzed by enzyme-linked immunosorbent assays (ELISA) for lipopolysaccharide, I-FABP, and D-lactate as well as flow cytometry for CD4 and $\mathrm{CD} 8$ cell counts.

The authors found several microbial genera depleted in the HIV-infected group, including Roseburia, Lachnospiraceae, Alistipes, and Ruminococcaceae, whereas Psychrobacter was increased. Of six stool SCFAs analyzed, valeric acid and butyric acid were decreased in the HIVinfected group, consistent with decreased abundance of butyrate-producing bacteria. Mild infiltration of inflammatory cells was seen in biopsies from the HIV-infected group, but claudin-1 expression was not significantly different. No between-group differences were seen for the mucosal marker of intestinal permeability claudin-1 or systemic markers I-FABP, D-lactate, and LPS.

The compositional differences in the gut microbiota and loss of butyrate-producing bacteria in HIV-infected subjects compared to HIV-negative controls reported by Qing et al. are, at least partially, consistent with those noted by other authors [8]. These findings suggest that the degree of variation in SCFA levels seen between HIV-infected and HIVnegative individuals is not adequate to induce differences in mucosal claudin-1 or serum markers of gut permeability. In the Qing study, comparisons between the HIV-infected and HIV-negative group are significantly limited by the difference in sexual orientation between the groups. Lack of detailed dietary information in the Qing study also adds the possibility that differences between groups were secondary to different diets.

The greatest strength of this study is the simultaneous assessment of multiple points along the microbiome-metabolome-gut barrier-systemic inflammation continuum. This allows a systems-based approach, which may improve the reliability of their findings. The lack of difference in systemic markers of gut integrity between the two groups is somewhat surprising given the published experience, but appears to be explained by the lack of gut barrier pathology noted on biopsy samples. Alternatively, a more comprehensive evaluation of mucosal $\mathrm{T}$ cell subsets may have identified differences as butyrate is hypothesized to induce mucosal Tregs in vivo [7]. Directly measuring paracellular permeability by absorption of simple sugars such as lactulose and mannitol may also have identified gut barrier alterations associated with depleted SCFAs. It remains unclear how mucosal proteins, such as mucosal claudin-1, correlate with dynamic tests of permeability such as simple sugar absorption or downstream inflammation, and clinical outcomes.

Their negative results are also unlikely to have been caused by the above-mentioned confounding factors, which might create spurious differences. Given the lack of published experience, it is difficult to know how to calculate statistical power for these measures as it is unclear how large a change in stool SCFA levels or epithelial tight junctional proteins is physiologically significant.

The authors suggest that these individuals may have been sampled too early in the course of HIV infection to find this pathology, but the median CD4 nadir of 135 argues otherwise. Depletion of CD4 cells from the intestinal compartment appears to occur long before depletion in the peripheral blood, so if mucosal damage is permanent, it should be present [3]. The null hypothesis, that gut microbiota composition and SCFA content of stool have little influence on gut epithelial tight junctions and systemic markers, should be considered.

Standardized antiretroviral therapy is rarely seen in HIV microbiome studies; antiretroviral regimens likely impact the gut environment in different ways. The inclusion of only those with NRTI/NNRTI regimens strengthens this study as it should limit variation within the HIV-infected group.

Data in the HIV and gut microbiome/metabolome field remain mostly descriptive. Longitudinal studies will be necessary to move the field past associations to more predictive models. Multiple sampling points such as stool, rectal swab, colonic mucosal biopsy, and small bowel aspiration or biopsy will need to be concurrently evaluated to determine which is most relevant to systemic disease. Currently, there are no data linking the gut microbiome in HIV patients, via any sampling methodology, to later clinical outcomes. Cross-sectional studies have suggested that the microbiota of mucosal biopsy samples is tightly correlated to serum markers of immune activation than that of stool samples, but it is not known whether this correlation is true for clinical outcomes [5].

Future studies will need to control for demographic differences. Detailed dietary information may explain changes in microbial composition and metabolism. Ultimately, descriptive studies such as this are limited by the inability to control for the myriad factors which may influence the gut microbiome and metabolome. Shotgun metagenomics would allow direct correlation of genes involved in SCFA synthesis with stool SCFA levels. More thorough investigation of colonic mucosa tissue samples may identify HIV-associated defects. 


\section{References}

1. Wada N, Jacobson LP, Cohen M, et al. Cause-specific life expectancies after 35 years of age for human immunodeficiency syndrome-infected and human immunodeficiency syndrome-negative individuals followed simultaneously in long-term cohort studies, 1984-2008. Am J Epidemiol. 2013;177:116-125.

2. Hunt PW, Sinclair E, Rodriguez B, et al. Gut epithelial barrier dysfunction and innate immune activation predict mortality in treated HIV infection. J Infect Dis. 2014;15:1228-1238.

3. Costiniuk CT, Angel JB. Human immunodeficiency virus and the gastrointestinal immune system: does highly active antiretroviral therapy restore gut immunity? Mucosal Immunol. 2012;5:596-604.

4. Noguera-Julian M, Rocafort M, Guillén Y, et al. Gut microbiota linked to sexual preference and HIV infection. EBioMedicine. 2016;5:135-146. https://doi.org/10.1016/j.ebiom.2016.01.032.

5. Liu J, Williams B, Frank D, Dillon SM, Wilson CC, et al. Inside out: HIV, the gut microbiome, and the mucosal immune system. J Immunol. 2017;198:605-614.

6. Plöger S, Stumpff F, Penner GB, et al. Microbial butyrate and its role for barrier function in the gastrointestinal tract. Ann NY Acad Sci. 2012;1258:52-59.
7. Dillon SM, Kibbie J, Lee EJ, et al. Low abundance of colonic butyrate-producing bacteria in HIV infection is associated with microbial translocation and immune activation. AIDS. 2017;31:511-521.

8. Hamer HM, Jonkers DM, Vanhoutvin SA, Troost FJ, Rijkers G, et al. Effect of butyrate enemas on inflammation and antioxidant status in the colonic mucosa of patients with ulcerative colitis in remission. Clin Nutr. 2010;29:738-744.

9. Serrano-Villar S, Vázquez-Castellanos JF, Vallejo A, Latorre A, Sainz T, et al. The effects of prebiotics on microbial dysbiosis, butyrate production and immunity in HIV-infected subjects. Mucosal Immunol. 2017;10:1279-1293.

10. Qing Y, et al. Gut microbiome, short-chain fatty acids, and mucosa injury in young adults with human immunodeficiency virus infection. Dig Dis Sci. (Epub ahead of print). https://doi.org/10.1007/ s10620-018-5428-2.

Publisher's Note Springer Nature remains neutral with regard to jurisdictional claims in published maps and institutional affiliations. 